\title{
Numerical and experimental assessment of turbulent kinetic energy in an aortic coarctation
}

\author{
Jonas Lantz, Tino Ebbers, Jan Engvall and Matts Karlsson
}

\section{Linköping University Post Print}

\section{Tweet}

N.B.: When citing this work, cite the original article.

Original Publication:

Jonas Lantz, Tino Ebbers, Jan Engvall and Matts Karlsson, Numerical and experimental assessment of turbulent kinetic energy in an aortic coarctation, 2013, Journal of Biomechanics, (46), 11, 1851-1858.

http://dx.doi.org/10.1016/j.jbiomech.2013.04.028

Copyright: Elsevier

http://www.elsevier.com/

Postprint available at: Linköping University Electronic Press

http://urn.kb.se/resolve?urn=urn:nbn:se:liu:diva-85683 


\title{
Numerical and Experimental Assessment of Turbulent Kinetic Energy in an Aortic Coarctation
}

\author{
Jonas Lantz ${ }^{\mathrm{a}}$, Tino Ebbers ${ }^{\mathrm{b}, \mathrm{c}}$, Jan Engvallc, Matts Karlsson ${ }^{\mathrm{a}}$ \\ ${ }^{a}$ Department of Management and Engineering \\ ${ }^{b}$ Department of Science and Technology \\ ${ }^{c}$ Department of Medical and Health Sciences \\ Linköping University \\ SE-581 83 Linköping \\ Sweden \\ jonas.lantz@liu.se
}

\begin{abstract}
The turbulent blood flow through an aortic coarctation was studied experimentally using magnetic resonance imaging (MRI), and numerically using computational fluid dynamics (CFD), before and after catheter intervention. Turbulent kinetic energy (TKE) was computed in the numerical model using large eddy simulation and compared with direct in vivo MRI measurements. Despite the two totally different methods to obtain TKE values, both quantitative and qualitative results agreed very well. The results showed that even though blood flow rate and Reynolds number increased after intervention, total turbulent kinetic energy levels decreased in the coarctation. Therefore, the use of the Reynolds number alone as a measure of turbulence in cardiovascular flows should be used with caution. Furthermore, the change in flow field and kinetic energy were assessed, and it was found that before intervention a jet formed in the throat of the coarctation, which impacted the arterial wall just downstream the constriction. After intervention the jet was significantly weaker and broke up almost immediately, presumably resulting in less stress on the wall. As there was a good agreement between measurements and numerical results (the increase and decrease of integrated TKE matched measurements almost perfectly while peak values differed by approximately $1 \mathrm{~mJ}$ ), the CFD results confirmed the MRI measurements while at the same time providing high-resolution details about the flow. Thus, MR-based TKE measurements might be useful as a diagnostic tool when evaluating intervention outcome, while the detailed numerical results might
\end{abstract}


be useful for further understanding of the flow for treatment planning.

Keywords: Computational fluid dynamics, Large eddy simulation, Magnetic resonance imaging, Turbulent flow

\section{Introduction}

Aortic coarctation is a congenital disease where the aorta is narrowed, impairing blood flow to downstream blood vessels and organs. Pathologic features include cardiac hypertrophy and degenerative changes in the proximal aorta (Nichols et al., 2011), and increased blood pressure in the upper part of the body because of the small volume receiving the full stroke volume. The severity of the coarctation before as well as after intervention is usually estimated by a catheter measurement of the blood pressure gradient across the coarctation. The narrowing of the aorta creates a flow jet with high velocity, inducing a very complex turbulent flow field. It has been suggested that morbidity among patients with aortic coarctation can be explained by altered hemodynamics and vascular biomechanics (O'Rourke and Cartmill, 1971). Recently, researchers has characterized changes of hemodynamic parameters such as pulse blood pressure, aortic capacitance, and wall shear stress due to the presence of an aortic coarctation (Frydrychowicz et al., 2008; LaDisa et al., 2011a,b; Coogan et al., 2012). Hemodynamic changes caused by the coarctation can result in endothelial dysfunction, dedifferentiation of arterial smooth muscle, and medial thickening (Menon et al., 2012).

In order to assess the flow, MRI (magnetic resonance imaging) measurements can give information on the flow field and it has recently been shown that it is possible to estimate the turbulent kinetic energy (TKE) using MRI (Dyverfeldt et al., 2008). Numerical modeling with computational fluid dynamics (CFD) can provide additional insights at a significantly higher resolution compared to MRI. Besides what-if scenarios and intervention planning, high-resolution details of the flow can be investigated as the resolution in CFD, in essence, is limited only by computer power. Accurate treatment of boundary conditions and turbulent flow must be considered to obtain reliable results.

In an aortic coarctation, the blood flow can transition from a well structured laminar state to a chaotic turbulent state, which is challenging to model. A common approach used in engineering applications is to use a 
RANS-model (Reynolds-Averaged Navier-Stokes), where the effect of the turbulent fluctuations on the mean flow are modeled through different turbulence models. As noted in a review article by Yoganathan et al. (2005), RANS models are not the ideal choice for disturbed cardiovascular flows, but instead, a scale-resolving turbulence model such as large eddy simulation (LES) would be more suitable, due to its ability to resolve velocity fluctuations and to handle transition to turbulence. There have been a number of studies using LES on idealized blood vessels with a constriction (Mittal et al., 2003; Paul et al., 2009; Gårdhagen et al., 2010), and very good agreement compared to experimental results has been found, demonstrating the potential for modeling both physiological and pathological low-Reynolds number turbulent flows with LES.

Olivieri et al. (2011) computed steady flow in native and surgically repaired aortic arches in order to correlate hemodynamic indices with the incidence of late morbidity. They found that varying patterns of wall shear stress as a result from abnormal wall remodeling may be involved in clinical vascular dysfunction. The effect on cardiac work load when virtually removing an coarctation was investigated by Kim et al. (2009). They used a deformable wall with uniform properties throughout the domain, and a lumped parameter heart model to account for the changes in velocity and pressure before and after intervention. They found a reduction in the afterload on the left ventricle after intervention, as blood pressure levels decreased.

Recently, Arzani et al. (2011) simulated the flow through an aortic coarctation with rigid walls using a second-order accurate direct numerical simulation, with the purpose of validating numerical predictions of TKE with in vivo MRI measurements. Their results showed a good agreement between measurements and numerical results, with an relative difference on the order of $10 \%$.

In this study, the flow through an aortic coarctation in a 63 year old female was studied. Balloon dilatation without stenting was performed to increase the diameter and catheter measurements showed a decrease in pressure drop after intervention, with increased blood flow through the coarctation as a result. Therefore, two cases were studied using CFD: blood flow through the coarctation before and after intervention, from hereon denoted as preintervention and post-intervention. The goal of this study was to resolve the flow features, and to compare the kinetic and turbulent kinetic energy obtained in the numerical simulations to MRI measurements. A long-term goal would be to use flow features derived from measurement techniques 
or numerical simulations, or a combination thereof, as an aid in diagnosis, evaluation and treatment planning of aortic coarctations.

\section{Method}

Here a short overview of the method is described; details on the CFD model and MRI acquisition are given in the supplementary materials. For $\mathrm{CFD}$, the model included the ascending aorta, two arteries leaving the aortic arch, an artery leaving the aorta in the vicinity of the coarctation, and the descending and thoracic aorta, see Figure 1. Geometry and inflow boundary profiles were obtained from MRI data. MRI was also used to directly measure the time-resolved velocity and TKE field in the 3D volume encompassing these vessels.

\subsection{Numerical Model}

A scale-resolving turbulence model, LES, was employed to resolve the turbulent features in the flow. The WALE subgrid model was used to handle the turbulent scales smaller than the grid size, while the larger scales were resolved. Details on the turbulence model were described in earlier work (Lantz and Karlsson, 2011). The simulations were carried out using ANSYS CFX 14.0. Velocity profiles measured by MRI were prescribed in the ascending aorta, providing physiologically accurate inlet boundary conditions, while measured mass flow rates were specified in the two vessels leaving the aortic arch. A pressure boundary condition was set in the descending aorta. The mesh sizes were on the order of 7 Million anisotropic hexahedral cells, with finer resolution near the walls and in the immediate post-stenotic region where most turbulent structures were found. Mesh independency tests and two-point correlations were carried out to ensure that the mesh resolution was fine enough, see supplementary materials for details. Phase averages of the velocity field were computed in the CFD simulation, and it was found that 12 cardiac cycles were needed to ensure statistically convergent results. A comparison between LES and simpler RANS turbulence models was performed, see the supplementary materials.

Using the Reynolds decomposition to decompose the velocity signal $u$ into a mean $\bar{u}$ and a fluctuating component $u^{\prime}$, the amount of turbulent fluctuations can be described by the root-mean-square of the difference of instantaneous velocity and mean velocity (Pope, 2000; Versteeg and Malalasekera, 
2007), as:

$$
u^{\prime}=\sqrt{\frac{1}{N} \sum_{i=1}^{N}\left(u_{i}-\bar{u}\right)^{2}}
$$

where $N$ is the number of samples in the signal. It is then possible to define the turbulent kinetic energy, as:

$$
\mathrm{TKE}=\frac{1}{2} \rho\left(u^{\prime 2}+v^{\prime 2}+w^{\prime 2}\right)
$$

where $u^{\prime}, v^{\prime}$, and $w^{\prime}$ are the fluctuating velocity components and $\rho$ the density. As a comparison, the kinetic energy is defined as:

$$
\mathrm{KE}=\frac{1}{2} \rho\left(u^{2}+v^{2}+w^{2}\right)
$$

where $u, v, w$ are the phase-averaged velocity components.

\subsection{MRI acquisition and segmentation}

Experimental data for the numerical model as well as direct measurements were obtained using a $1.5 \mathrm{~T}$ Philips Achieva MRI scanner (Philips Health Care, Best, the Netherlands). The geometry of the aorta was obtained within a breath hold using a 3D gradient-echo sequence and subsequently segmented using a 3D level set algorithm implemented into a cardiac image analysis software package (Heiberg et al., 2010). The segmentation software gave a STL-representation of the inner aortic wall which set the boundary for the fluid domain.

Inflow boundary conditions were obtained in the ascending aorta using a 2D through-plane phase-contrast MRI measurement placed supracoronary perpendicular to the flow direction. The data were acquired within an expiration breath hold using retrospective cardiac gating to a vector cardiogram, and was reconstructed to 40 time-frames per heart cycle with a spatial resolution of $1.56 \times 1.56 \mathrm{~mm}$.

Direct measurements of the velocity and TKE in the whole aorta were obtained using time-resolved three-directional phase-contrast MRI. Full coverage of the complete cardiac cycle was obtained by retrospective VCG gating. Data were acquired during free breathing using a respiratory navigator-gated gradient-echo pulse sequence. The standard deviation of the velocity distribution for each component within a voxel is computed from the magnitude 
of the MRI signal, assuming a Gaussian velocity distribution, as described by Dyverfeldt et al. (2006). The TKE is computed from the intravoxel standard deviation in three mutually perpendicular directions (Dyverfeldt et al., 2008). Two scans with different motion encoding strengths were obtained to acquire velocity and TKE data. Both measurements were performed using a motion encoding scheme that included a reference flow-encoding segment with nulled motion sensitivity, enabling optimal data quality in the TKE estimation (Dyverfeldt et al., 2011).

The velocity and TKE data were reconstructed into 40 time frames per cardiac cycle and were corrected for phase-wraps and background phase errors due to concomitant gradient fields and eddy currents on the scanner. While TKE was directly measured in the scanner, KE was computed from the measured velocity 3D-field.

\section{Results}

After the intervention the cross-sectional area of the coarctation increased from 106 to $145 \mathrm{~mm}^{2}$, which decreased the pressure drop, yielding a higher mass flow rate. The blood pressure was measured with a catheter and before intervention it was $135 / 44 \mathrm{mmHg}$ proximal and $113 / 37 \mathrm{mmHg}$ distal the coarctation, i.e. the peak systolic pressure difference was $22 \mathrm{mmHg}$. After intervention the blood pressure was 132/46 proximal and 124/48 distal the coarctation, i.e. there was a remaining peak systolic pressure difference of $8 \mathrm{mmHg}$, which was considered acceptable. The flow rate in the ascending and descending aorta were measured with MRI, and are presented in Figure 2. The cardiac output changed from 4.5 to $5.2 \mathrm{~L} / \mathrm{min}$ in the ascending aorta, while it changed from 3.1 to $3.5 \mathrm{~L} / \mathrm{min}$ in the descending aorta. The Reynolds number is often used in order to quantify the amount of turbulence in a flow; for steady flow in a circular pipe a transition to turbulence is generally accepted to occur around $R e=2300$, while for pulsatile flows the critical value is normally higher (Peacock et al., 1998). As a result of the changed flow rate and diameter, the peak Reynolds number in the coarctation increased slightly from 7700 to about 8000 after intervention, see Figure 3.

The turbulent kinetic energy was computed for both cases and compared to MRI measurements. A point-by-point comparison between CFD and MRI results was not possible to do, as there was a significant difference in resolution; the number of MRI voxels used in the integration were approximately 2500 (isotropic) while about 3185000 anisotropic mesh cells 
were used in the CFD model, i.e. there was more than a 1200 -fold difference in resolution. Integrated values of TKE in a volume downstream the coarctation were computed, and a good agreement between the numerical model and the measurements was obtained, see Figure 4.

For the pre-intervention model, the numerical model slightly underestimated the maximum TKE compared to MRI measurements (13\% difference in peak values), but matched the increase and decrease (the maximum difference between MRI and CFD were $<8 \%$ for the build-up slope and $<4 \%$ for the decay slope). There was a $0.03 \mathrm{~s}$ time difference between the build-up slopes in the MRI and CFD results while the decay slopes were practically on each other.

The post-intervention model showed a decrease in TKE, as a result of the increased coarctation diameter. Here, a larger difference between CFD and MRI derived TKE was obtained; the peak values differed by $1.5 \mathrm{~mJ}$ and $0.06 \mathrm{~s}$, and the build-up and decay slopes are not captured as well as in the pre-intervention case. The build-up slope of the CFD results is steeper than the MRI results, while the decay-slope for the CFD result agree well in shape with the MRI result, but is $0.03 \mathrm{~ms}$ earlier.

For both cases the increase in TKE start just after peak flow rate, with maximum values in the systolic deceleration phase. Turbulence levels were almost zero during systolic acceleration and peak systole, as acceleration tend to stabilize the flow (Peacock et al., 1998). Peak values and when they occurred during the cardiac cycle are indicated in Table. 1. Considering maximum values, the numerical simulation predicted a value of $1464 \mathrm{~Pa}$ while the measurement was slightly lower with $1315 \mathrm{~Pa}$ in the pre-intervention model. In the post-intervention model, the maximum values were $838 \mathrm{~Pa}$ and $723 \mathrm{~Pa}$ in the $\mathrm{CFD}$ and MRI results, respectively.

A volume rendering of the TKE is presented in Figure 5, and clearly show the details that are possible to obtain in CFD simulations compared to MRI measurements. Both the CFD and MRI results show how the fluctuations start in the aortic arch, in the vicinity of the branching vessels, i.e. a transition to turbulent flow is triggered before the coarctation. In order to explain why the flow was turbulent before the coarctation, it was decided to investigate the kinetic energy (KE) in addition to TKE along the geometrical centerline using data from both the CFD simulations and MRI measurements. The centerline starts in the ascending aorta and ends in the thoracic aorta, and if the kinetic and turbulent kinetic energy are plotted over time, a spatio-temporal map is created, see Figures 6 and 7. The kinetic energy is 
low in the ascending aorta as a result of the large cross-sectional area that yields a lower velocity magnitude. In the upper part of the aortic arch and beginning of descending aorta (denoted LCC in the figure) the flow makes a sharp turn, which locally increases the kinetic energy as the fluid accelerates. This is most evident in the post-intervention case and could be a result of the intervention. Shortly after, the KE levels decrease until the coarctation, where, as expected, the largest values are found. It is also evident from both the CFD and MRI results that the KE decreased in the coarctation after intervention. High levels of $\mathrm{KE}$ are present from peak systole to maximum deceleration, and then decrease rapidly.

Considering turbulent effects, there are practically no TKE along the centerline in the ascending aorta and aortic arch in the CFD results, while the MRI results indicate some degree of velocity fluctuations. This can be due to noise in the measurements or caused by instabilities created by the aortic valve, which was not included in the CFD model. However, turbulent fluctuations become apparent directly after the sharp turn in the end of the aortic arch in both the simulations and measurements. Note that the TKE levels increase after peak systole, i.e. in the deceleration phase and not at peak flow rate. In the throat of the coarctation, the flow shows a tendency to relaminarize as TKE levels decrease while KE increase. Directly after the coarctation the TKE increase a second time as the flow again becomes turbulent. From Figure 7 it seems that the largest TKE levels are found in the post-intervention case. However, while this is true for the centerline, the total TKE level in the downstream region of the coarctation decreases after intervention, as previously shown in Figure 4. The increase of TKE along the centerline can be explained by investigating how the intervention changed the flow field; not only did the diameter increase, the jet emanating from the coarctation also changed direction after intervention. To find high speed regions in the flow domain, iso-volumes with velocity magnitude $>1 \mathrm{~m} / \mathrm{s}$ were post-processed. A seen in Figure 8, at peak flow rate a jet forms in the coarctation which hits the arterial wall in the pre-op case. After intervention the coarctation is wider and while there is a tendency to jet formation, the flow is now directed to the center of the vessel. In addition, as the velocity in the post-intervention jet is lower (even though mass flow rate is higher), the kinetic energy is lowered, facilitating breaking up the jet by the surrounding fluid. As the jet is more centered in the vessel after intervention, TKE levels will increase locally along the centerline, but the total level downstream the coarctation is still decreased. 
Comparing the KE and TKE levels, it can be seen that the KE is about an order of magnitude larger than the TKE, but due to the high velocities created in the coarctation, turbulent kinetic energy on the order of $100 \mathrm{~Pa}$ are present far down in the descending aorta in both cases.

\section{Discussion}

In this study, the turbulent flow field in an aortic coarctation was investigated before and after catheter intervention. MRI measurements showed that cardiac output increased after intervention, due to a decrease in pressure drop over the coarctation. The flow rate increased slightly more in the ascending aorta than in the descending aorta, which may be explained by a change in resistance of collateral vessels. As the pressure drop decreased, so did also left ventricular work load, which is beneficial for the patient (Coogan et al., 2012). However, it is likely that the increase in area of the studied coarctation was insufficient to achieve an optimal effect on $24 \mathrm{hr}$ ambulatory blood pressure in the upper part of the body (Engvall and Nyström, 2001).

Turbulent kinetic energy was used to quantify the turbulence levels using both MRI measurements and numerical models. The energy in turbulent flows and anisotropy effects are predominantly present in large-scale motion (Pope, 2000), and it should therefore be possible to estimate TKE with the resolution found in MRI. Both the CFD and MRI results showed that integrated TKE levels decreased after intervention. The computational results also showed that the jet forming in the throat of the coarctation hits the distal arterial wall in the pre-op case, which may lead to remodeling of the aortic wall (Nichols et al., 2011). This is e.g. also seen in patients with a bicuspid aortic valve, where a jet forms in the aortic valve and hits the ascending aorta, (Della Corte et al., 2007; Schaefer et al., 2008). This could provide an explanation to why some patients experience post-stenotic dilatation directly after a coarctation. In this case, after intervention the jet was directed towards the center of the cross-section of the aorta and broke easily up due to the decrease in kinetic energy.

The volume renderings and integrated TKE levels indicated that the turbulent fluctuations decreased after intervention, but they did not disappear. Interestingly, transition to turbulence was triggered not only at the throat of the coarctation, but also earlier in the aortic arch close to the branching vessels where the aortic geometry forced the blood flow to take accelerate and make a sharp turn. The total kinetic energy of the flow is the sum of the 
KE and TKE, and regions with elevated KE could, presumably, also contain elevated TKE values. This was evident by the results in Figures 6 and 7, where elevated $\mathrm{KE}$ values corresponded to the onset of elevated TKE values. But, while elevated KE regions are well defined in space and time (e.g. in the vicinity of the coarctation between the PS and ES time points in Figure 6), turbulent regions are not; TKE is produced in the high shear flow in and distal the coarctation (and convected with the flow), which creates a large irregular region with elevated TKE values as seen in Figure 7. In addition, the centerline results enable a qualitative comparison of TKE between the CFD and MRI results (where local and instantaneous values are used instead of integrated values). The agreement seems to be good, with low values in the ascending aorta and a transition to turbulence before the coarctation.

There are studies that have tried to correlate the Reynolds number in the aorta to flow instabilities and turbulence, see e.g. Stalder et al. (2011). In this study, the Reynolds number in the coarctation increased after intervention, while at the same time, both CFD and MRI results showed a decrease of TKE downstream the coarctation. This raises the question whether the Reynolds number by itself is a suitable measure of the amount of turbulence in the flow, at least under pulsatile flow conditions in vascular geometries. Instead, flow features such as velocity fluctuations may be used, e.g. quantified by TKE. Note, however, that this is a single measurement in one patient, and larger studies need to be performed to achieve a statistically significant result.

There are of course inherent limitations in both the numerical model and the MRI measurements. The CFD model assumes rigid walls, which, in practice will give unrealistic mass- and pressure wave speeds. The compliance of the wall might also affect the flow field, but it was not taken into account. Errors introduced from segmentation of MRI images and uncertainties in the boundary conditions may also influence the accuracy of the numerical model. Additionally, the potential influence of inflow effects from collateral vessels was ignored. The MRI measurements correlate the signal loss to the amount of flow disturbances within each voxel. Inherent signal noise and a MRI resolution on the order of millimeters, which enables the resolution of only the largest turbulent scales, may affect the accuracy of the TKE estimation.

The turbulent fluctuations were computed by two totally different methods, and despite the possible error sources, the comparison between MRImeasured and numerically resolved KE and TKE agreed very well. This indicates that the measurements are reliable and reproducible, and might be used as a complement to traditional procedures when evaluating the out- 
come of an intervention. The numerical model can provide further insights and details about the flow field that is not possible by the use of MRI. If TKE would be used in clinical practice, the peak values or the volume of elevated values above a certain threshold might be most useful for the physician, as high or increased values of TKE indicates an abnormal or pathophysiological flow situation.

\section{Acknowledgments}

This work was supported by a grant from the Swedish research council, VR 2010-4282. The Swedish National Infrastructure for Computing (SNIC) is acknowledged for computational resources provided by the National Supercomputer Centre (NSC) and the High Performance Computing Center North (HPC2N) under grant No. SNIC022/09-11. This work has been conducted in collaboration with the Center for Medical Image Science and Visualization (CMIV, http://www.cmiv.liu.se/) at Linköping University, Sweden. CMIV is acknowledged for provision of financial support and access to leading edge research infrastructure.

\section{Conflict of Interest Statement}

None of the authors have any commercial or non-commercial relationship that might lead to a conflict of interest.

\section{References}

Arzani, A., Dyverfeldt, P., Ebbers, T., Shadden, S., 2011. In vivo validation of numerical prediction for turbulence intensity in an aortic coarctation. Annals of Biomedical Engineering , 1-11.

Coogan, J.S., Humphrey, J.D., Figueroa, C.A., 2012. Computational simulations of hemodynamic changes within thoracic, coronary, and cerebral arteries following early wall remodeling in response to distal aortic coarctation. Biomechanics and Modeling in Mechanobiology , 1-15.

Della Corte, A., Bancone, C., Quarto, C., Dialetto, G., Covino, F., Scardone, M., Caianiello, G., Cotrufo, M., 2007. Predictors of ascending aortic dilatation with bicuspid aortic valve: a wide spectrum of disease expression. European Journal of Cardio-Thoracic Surgery 31, 397-405. 
Dyverfeldt, P., Kvitting, J., Sigfridsson, A., Engvall, J., Bolger, A., Ebbers, T., 2008. Assessment of fluctuating velocities in disturbed cardiovascular blood flow: In vivo feasibility of generalized phase-contrast mri. Journal of Magnetic Resonance Imaging 28, 655-663.

Dyverfeldt, P., Sigfridsson, A., Knutsson, H., Ebbers, T., 2011. A novel mri framework for the quantification of any moment of arbitrary velocity distributions. Magnetic Resonance in Medicine 65, 725-731.

Dyverfeldt, P., Sigfridsson, A., Kvitting, J., Ebbers, T., 2006. Quantification of intravoxel velocity standard deviation and turbulence intensity by generalizing phase-contrast mri. Magnetic resonance in medicine 56, 850-858.

Engvall, J., Nyström, F., 2001. Daytime ambulatory blood pressure correlates strongly with the echocardiographic diameter of aortic coarctation. Scandinavian cardiovascular journal: SCJ 35, 335.

Frydrychowicz, A., Arnold, R., Hirtler, D., Schlensak, C., Stalder, A.F., Hennig, J., Langer, M., Markl, M., 2008. Multidirectional flow analysis by cardiovascular magnetic resonance in aneurysm development following repair of aortic coarctation. Journal of Cardiovascular Magnetic Resonance 10,30 .

Gårdhagen, R., Lantz, J., Carlsson, F., Karlsson, M., 2010. Quantifying turbulent wall shear stress in a stenosed pipe using large eddy simulation. Journal of biomechanical engineering 132, 061002.

Heiberg, E., Sjogren, J., Ugander, M., Carlsson, M., Engblom, H., Arheden, H., 2010. Design and validation of Segment-freely available software for cardiovascular image analysis. BMC Med Imaging 10, 1.

Kim, H., Vignon-Clementel, I., Figueroa, C., LaDisa, J., Jansen, K., Feinstein, J., Taylor, C., 2009. On coupling a lumped parameter heart model and a three-dimensional finite element aorta model. Annals of biomedical engineering 37, 2153-2169.

LaDisa, J.J., Alberto, F.C., Vignon-Clementel, I.E., Jin, K.H., Xiao, N., Ellwein, L.M., Chan, F.P., Feinstein, J.A., Taylor, C.A., et al., 2011a. Computational simulations for aortic coarctation: representative results from a sampling of patients. Journal of biomechanical engineering 133, 091008. 
LaDisa, J.J., Dholakia, R.J., Figueroa, C.A., Vignon-Clementel, I.E., Chan, F.P., Samyn, M.M., Cava, J.R., Taylor, C.A., Feinstein, J.A., 2011b. Computational simulations demonstrate altered wall shear stress in aortic coarctation patients treated by resection with end-to-end anastomosis. Congenital heart disease 6, 432-443.

Lantz, J., Karlsson, M., 2011. Large eddy simulation of LDL surface concentration in a subject specific human aorta. Journal of Biomechanics 4, $537-542$.

Menon, A., Eddinger, T.J., Wang, H., Wendell, D.C., Toth, J.M., LaDisa, J.F., 2012. Altered hemodynamics, endothelial function, and protein expression occur with aortic coarctation and persist after repair. American Journal of Physiology-Heart and Circulatory Physiology 303, H1304H1318.

Mittal, R., Simmons, S.P., Najjar, F., 2003. Numerical study of pulsatile flow in a constricted channel. Journal of Fluid Mechanics 485, 337-378.

Nichols, W., O’Rourke, M., Vlachopoulos, C., Arnold P. Hoeks, P., 2011. McDonald's Blood Flow in Arteries: Theoretical, Experimental and Clinical Principles. Hodder Arnold Publishers.

Olivieri, L., de Zélicourt, D., Haggerty, C., Ratnayaka, K., Cross, R., Yoganathan, A., 2011. Hemodynamic modeling of surgically repaired coarctation of the aorta. Cardiovascular Engineering and Technology , 1-8.

O'Rourke, M., Cartmill, T., 1971. Influence of aortic coarctation on pulsatile hemodynamics in the proximal aorta. Circulation 44, 281-292.

Paul, M.C., Mamun Molla, M., Roditi, G., 2009. Large-Eddy simulation of pulsatile blood flow. Med Eng Phys 31, 153-159.

Peacock, J., Jones, T., Tock, C., Lutz, R., 1998. The onset of turbulence in physiological pulsatile flow in a straight tube. Experiments in fluids 24, $1-9$.

Pope, S., 2000. Turbulent flows. Cambridge University Press.

Schaefer, B., Lewin, M., Stout, K., Gill, E., Prueitt, A., Byers, P., Otto, C., 2008. The bicuspid aortic valve: an integrated phenotypic classification of leaflet morphology and aortic root shape. Heart 94, 1634. 
Stalder, A., Frydrychowicz, A., Russe, M., Korvink, J., Hennig, J., Li, K., Markl, M., 2011. Assessment of flow instabilities in the healthy aorta using flow-sensitive mri. Journal of Magnetic Resonance Imaging 33, 839-846.

Versteeg, H., Malalasekera, W., 2007. An introduction to computational fluid dynamics: the finite volume method. Prentice Hall.

Yoganathan, A.P., Chandran, K.B., Sotiropoulos, F., 2005. Flow in prosthetic heart valves: state-of-the-art and future directions. Ann Biomed Eng 33, 1689-1694. 
Table 1: Computed and measured integrated TKE values for the pre- and post-intervention cases.

\begin{tabular}{llll} 
Pre & \multicolumn{2}{l}{ Post } \\
CFD & MRI & CFD & MRI \\
\hline $6.01 \mathrm{~mJ}(0.29 \mathrm{~s})$ & $6.95 \mathrm{~mJ}(0.28 \mathrm{~s})$ & $5.81 \mathrm{~mJ}(0.22 \mathrm{~s})$ & $4.26 \mathrm{~mJ}(0.28 \mathrm{~s})$
\end{tabular}




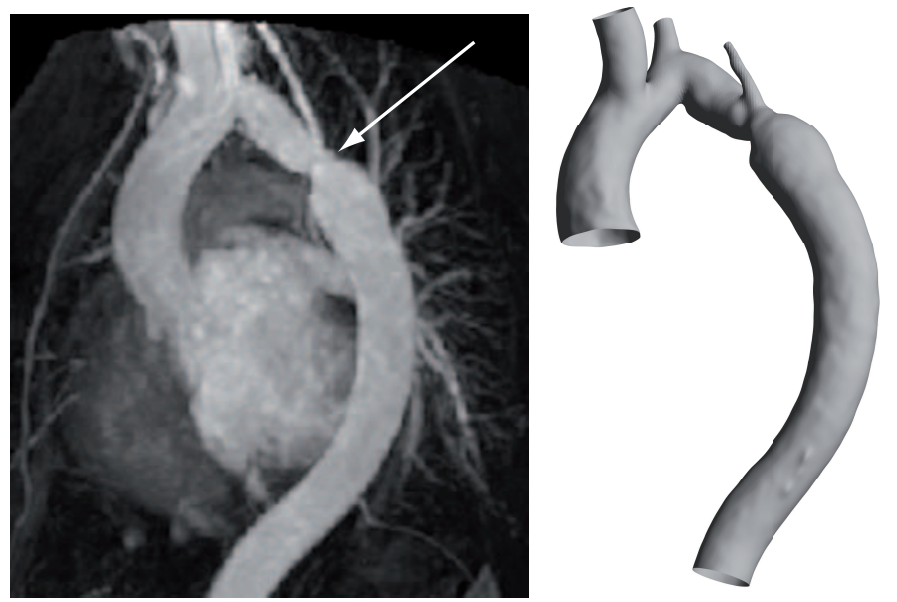

Figure 1: Left: Maximum Intensity Projection (MIP) image of the heart and aorta. The white arrow indicates the location of the aortic coarctation. Right: CAD surface of the aortic geometry after intervention. Notice that the vessel still is constricted and that there is a collateral vessel that originates from one of the carotid arteries and connects to the aorta in the vicinity of the coarctation. 

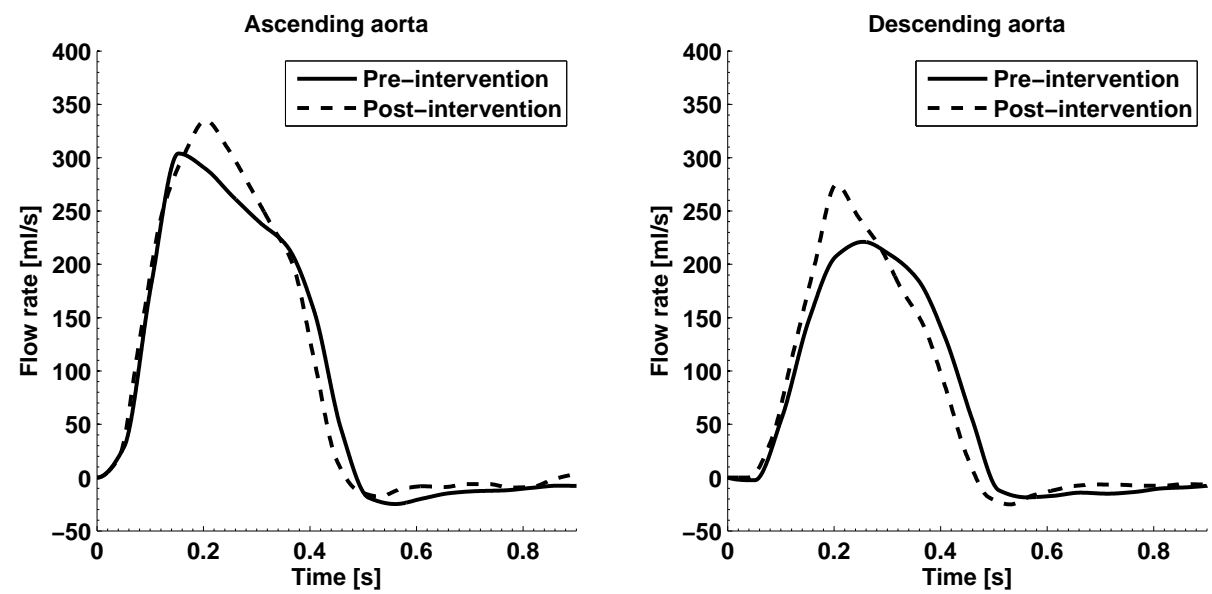

Figure 2: Measured flow rates in the ascending and descending aorta before and after intervention. Notice how the peak flow increases after intervention.

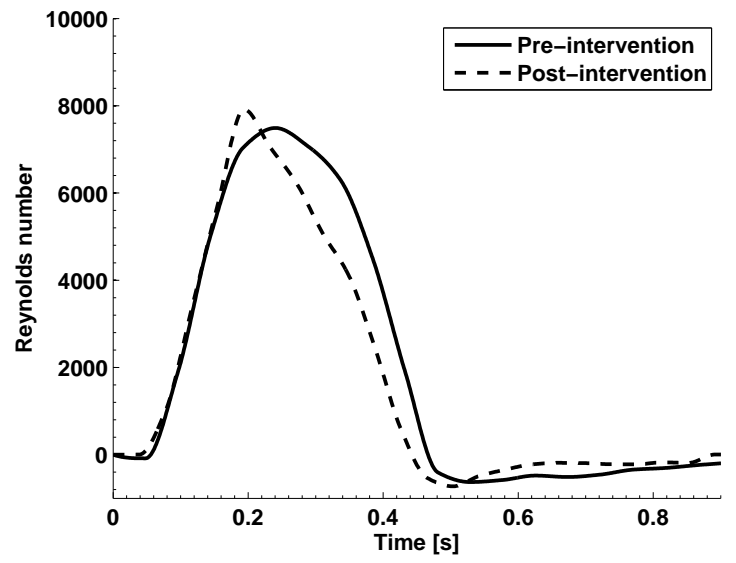

Figure 3: The Reynolds number in the throat of the coarctation during the cardiac cycle before and after intervention. Notice that the peak Reynolds number actually increases after intervention, as a result of the increased mass flow rate. 

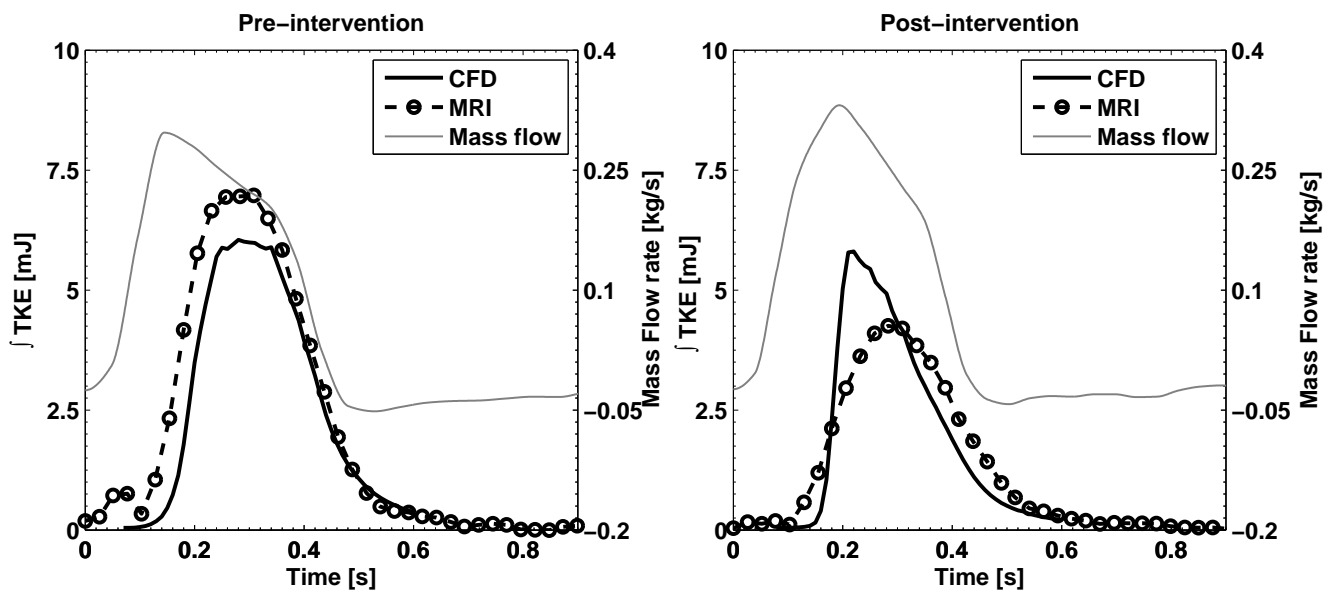

Figure 4: Left y-axis: Volume integral of TKE in the region downstream the coarctation (solid and dashed lines). Circles indicate temporal MRI resolution. Right y-axis: Measured mass flow rate in the ascending aorta, for reference (light gray line). The amount of voxels inside the volumes used for integration were approximately 10x10x25 (= 2500) for the MRI results and 70x70x650 (= 3185000) for the CFD simulation, i.e. about a 1200-fold greater spatial resolution overall. Volume where TKE is integrated is shown below the plots. 

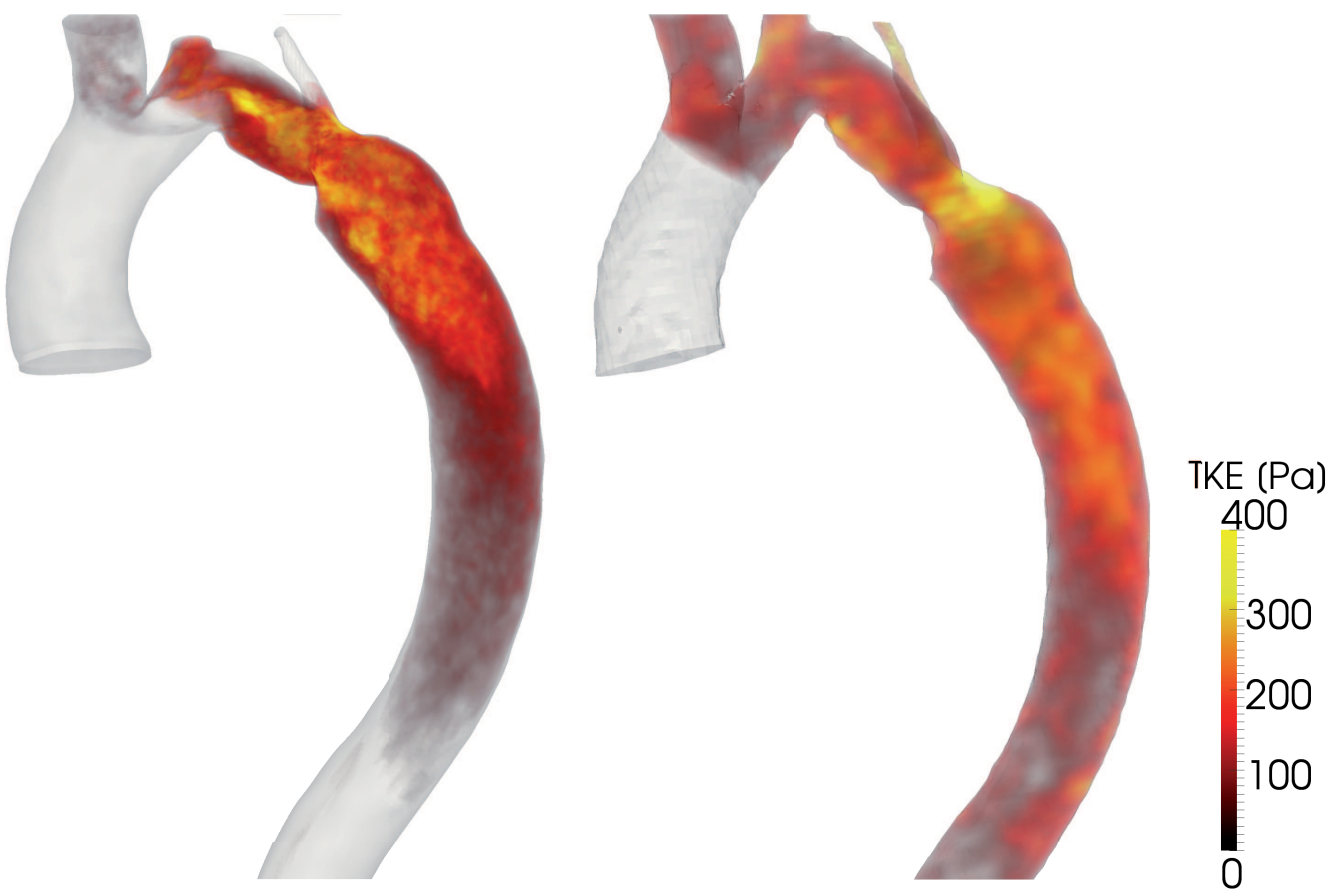

Figure 5: Volume rendering of TKE in the coarctation for the post-intervention model, at $t=0.35 \mathrm{~s}$. Left model is the CFD model, right is MRI measurements. 


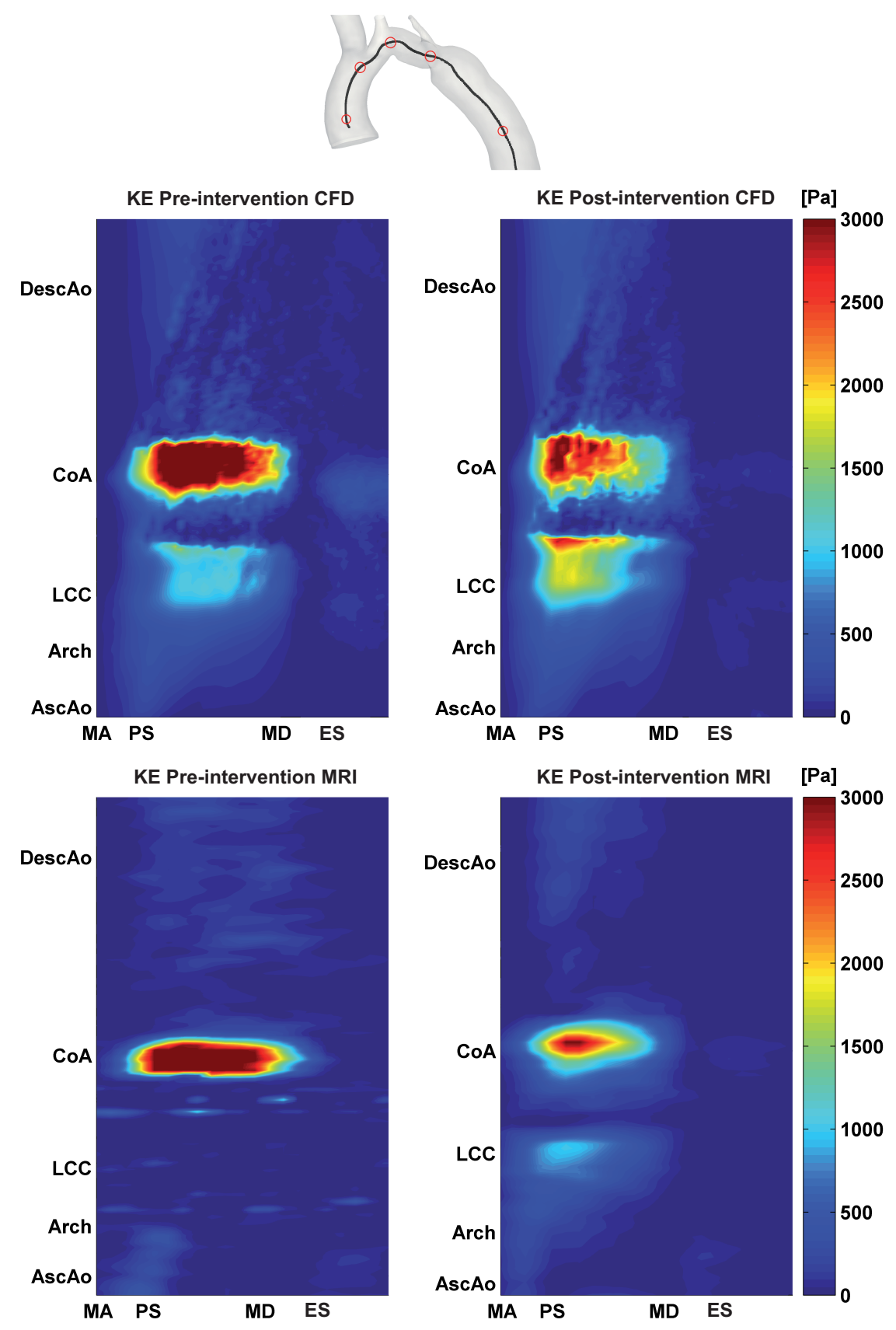

Figure 6: Kinetic energy along the centerline (y-axis) as a function of time (x-axis) in the pre-op and post-intervention cases. Top row is CFD results, lower row is MRI measurements. X-axis labels reads MA: maximum acceleration, PS: peak systole, MD: maximum deceleration, ES: end of systole. Y-axis locations are indicated in the figure above, and reads AscAo: ascending aorta, Arch: aortic arch, LCC: left common carotid artery, CoA: coarctation of the aorta, and DescAo: descending aorta. 


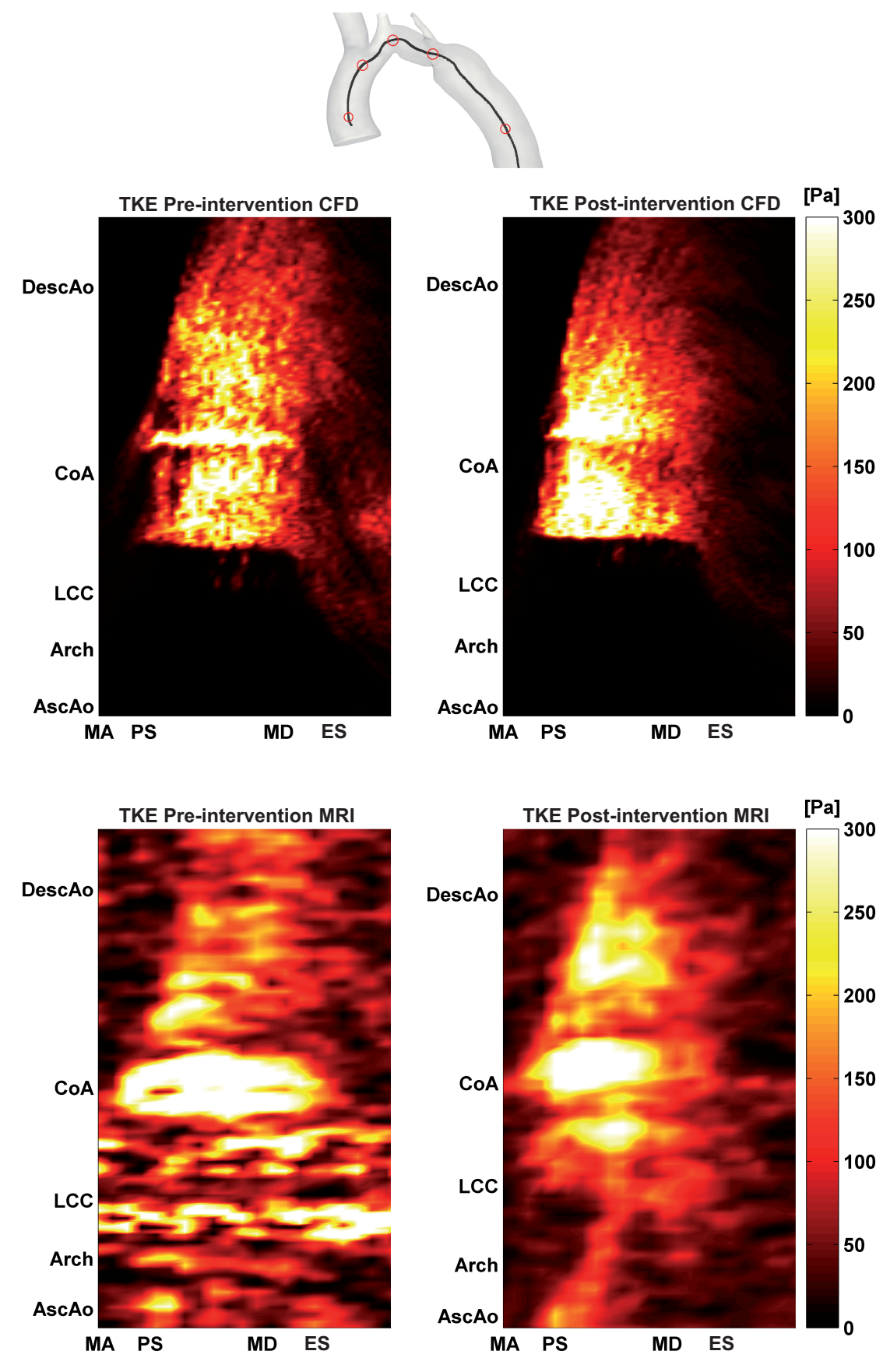

Figure 7: Turbulent kinetic energy along the centerline (y-axis) as a function of time (xaxis) in the pre-op and post-intervention cases. Top row is CFD results, lower row is MRI measurements. For axis legend, see Figure 6. 


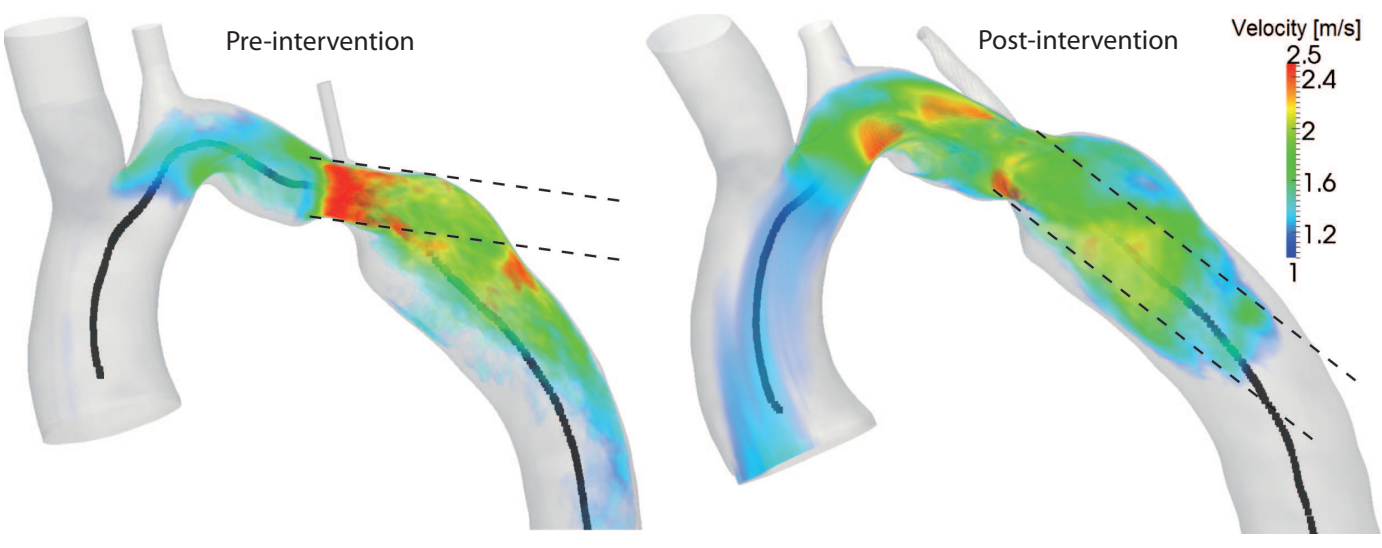

Figure 8: Volume rendering of velocity magnitude $>1 \mathrm{~m} / \mathrm{s}$. Solid line is the geometrical centerline and dashed lines show the direction of the jet emanating from the throat of the coarctation. Notice how the jet changes from hitting the wall in the pre-op case to break up in the center of the vessel in the post-intervention case. 